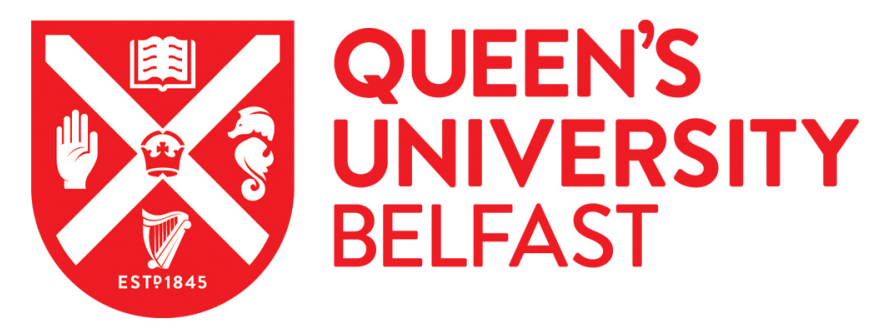

\title{
The Complex Solid-State Landscape of Sodium Diatrizoate Hydrates
}

Mohd Najib, M. N., Back, K., \& Edkins, K. (2017). The Complex Solid-State Landscape of Sodium Diatrizoate Hydrates. Chemistry - A European Journal, 23(68), 17339 -17347. https://doi.org/10.1002/chem.201703658

\author{
Published in: \\ Chemistry - A European Journal
}

\section{Document Version:}

Peer reviewed version

Queen's University Belfast - Research Portal:

Link to publication record in Queen's University Belfast Research Portal

\section{Publisher rights}

Copyright 2017 Wiley. This work is made available online in accordance with the publisher's policies. Please refer to any applicable terms of use of the publisher.

\section{General rights}

Copyright for the publications made accessible via the Queen's University Belfast Research Portal is retained by the author(s) and / or other copyright owners and it is a condition of accessing these publications that users recognise and abide by the legal requirements associated with these rights.

Take down policy

The Research Portal is Queen's institutional repository that provides access to Queen's research output. Every effort has been made to ensure that content in the Research Portal does not infringe any person's rights, or applicable UK laws. If you discover content in the Research Portal that you believe breaches copyright or violates any law, please contact openaccess@qub.ac.uk. 


\title{
The Complex Solid-State Landscape of Sodium Diatrizoate
}

\section{Hydrates}

\author{
Mohd Nadzri Mohd Najib, ${ }^{a, b}$ Kevin Back ${ }^{c}$ and Katharina Edkins ${ }^{* a}$
}

\begin{abstract}
Pharmaceutical sodium salts are prone to incorporate water into their crystal structure. The model compound diatrizoic acid monosodium salt, an X-ray contrast agent, has been investigated in depth towards its interaction with water in the solid state. Five hydrates with water content ranging from 0.3 to 8 molar equivalents of water show a high degree of interconvertibility, stoichiometric and non-stoichiometric behaviour, and potential of amorphisation during release of water. A DMSO/water mixed solvate further highlights the high attraction of this salt to incorporate water. All incorporated solvent coordinates to the sodium cation and can further interact and stabilise the respective crystal forms by hydrogen bonding. DTS thus highlights the importance of an in-depth investigation of sodium salts used pharmaceutically to guarantee dose uniformity and stability of final formulation.
\end{abstract}

\section{Introduction}

The challenge to optimize the physical and chemical properties of a new active pharmaceutical ingredient (API), such as flowability, hardness, stability and solubility, is an everyday challenge for the pharmaceutical industry. Many compounds as synthesized are non-optimal materials for manufacturing and patient safety, and thus further steps are taken, e.g. change of crystal form, cocrystallisation or salt formation. Especially for molecules showing low to very low solubility in water, salt formation is the most preferable strategy in the presence of an ionisable or protonatable moiety in the molecule. Traditionally, the salt-forming agents are chosen based on trial and error and the salt form selected by pharmaceutical chemists is based on a practical basis addressing cost and ease of production. ${ }^{[1]}$ However, in recent times, intelligent design for salt selection process is more favourable, ${ }^{[2]}$ and it is estimated that more than half of all drug molecules used in medicine are now marketed as salts. ${ }^{[3]}$ Overall, sodium salts are amongst the most abundant salt forms, only surpassed by hydrochloride salts of basic APIs. ${ }^{[4]}$

[a] Mohd Nadzri Bin Mohd Najib, Dr Katharina Edkins

School of Pharmacy

Queen's University Belfast

97 Lisburn Road, Belfast, BT9 7BL, UK

E-mail: K.Edkins@qub.ac.uk

[b] Mohd Nadzri Bin Mohd Najib

School of Medicine, Pharmacy and Health

Durham University

University Boulevard, Stockton-on-Tees, TS17 6BH, UK

[c] Dr Kevin Back

Pfizer Ltd.

Sandwich, CT13 9ND, UK

Supporting information for this article is given via a link at the end of the document
Connected to the improved water solubility, salts, and especially sodium salts, show hygroscopicity ${ }^{[5]}$ and an increased possibility of the formation of hydrated crystal forms. ${ }^{[1,4 a, 6]}$ These modifications include water molecules as an integral part of the crystal lattice. ${ }^{[7]}$ Due to thermodynamic reasons, hydrates have the lowest solubility in water of all solid forms of a specific compound, ${ }^{[6]}$ and thus potentially counteract the benefits of the initial salt formation. In addition, the presence of water in the crystal lattice has been shown to increase molecular mobility and lead to a higher reaction rate in the solid state, leading to enhanced chemical degradation of drug compounds. ${ }^{[8]}$ Crystalline hydrates are classified into three groups: isolated site hydrates incorporate the water molecules individually or in small clusters, whilst channel hydrates have the structural feature of water-filled pores or sheets. ${ }^{[9]}$ The third class of metal-associated hydrates is especially important for pharmaceutical sodium salts, as the water molecules coordinate to metal centres in the crystal structure and the resulting hydrated crystal form can show high or very high stability against dehydration. ${ }^{[10]}$

Even though pharmaceutical sodium salts are well established, manifold challenges can occur during the manufacturing stage. Disproportionation of the salt into the neutral API and an inorganic sodium salt in the presence of water can cause stability issues of the API during formulation, ${ }^{[11]}$ as has been shown for furosemide salts ${ }^{[12]}$ as well as for ifetroban sodium. ${ }^{[13]}$ The opposite effect of spontaneous salt formation can be triggered by humidity within the final formulation: One example for this is piroxicam, which during wet granulation in the presence of basic salts such as sodium carbonate or calcium phosphate undergoes spontaneous salt formation. Whilst the dissolution behaviour of piroxicam is not impacted by this change in solidstate form, the closely related lornoxicam shows a considerable increase in solubility jeopardizing the patient's safety. ${ }^{[14]}$ However, the solid solution of amorphous indomethacine with its sodium salt revealed a substantial stabilizing effect of the salt on the amorphous material. ${ }^{[15]}$ This is particularly interesting in the case of very low solubility presented in drug compounds, as the amorphous form will have a higher solubility than any crystalline modification. ${ }^{[16]}$ Hydrated sodium salts can also offer an advantage from a manufacturing point of view; for example, diclofenac sodium tetrahydrate shows an improved flowability within the powder bed than its anhydrous counterpart, ${ }^{[17]}$ which simplifies industrial processes such as mixing, tableting and capsule filling.

The current European Pharmacopoeia 9.0 ${ }^{[18]}$ monographs 79 pharmaceutically active small organic compounds as their 


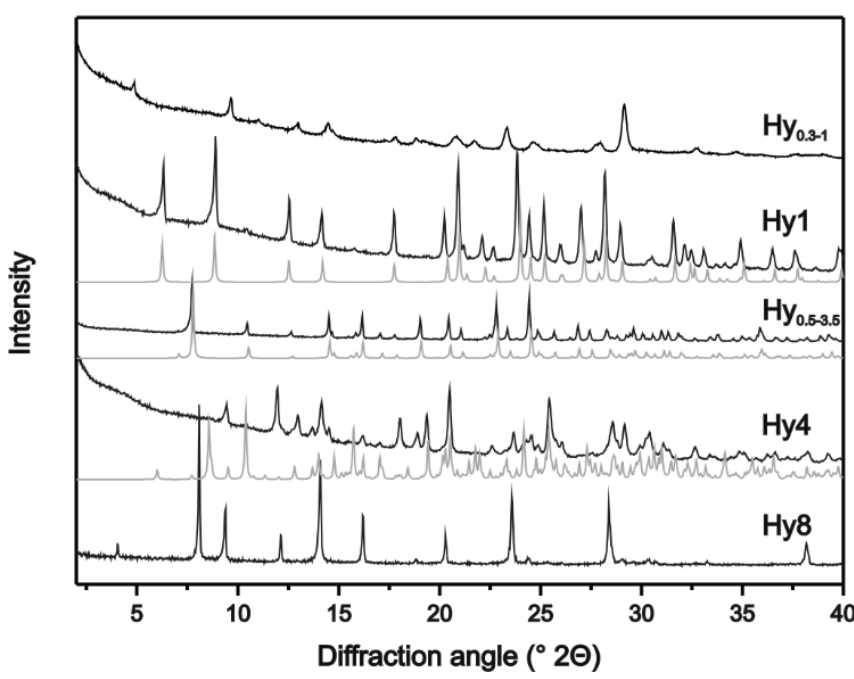

Figure 1. Powder X-ray diffraction (PXRD) trace of the different DTS hydrate forms. Experimental patterns in black, patterns calculated from single crystal structures in grey.

sodium salt, of which 13 show such high stability and/or prevalence that the hydrate is individually monographed or even the solely monographed material.

Due to their enhanced interaction with water, sodium salts can form multiple hydrated crystal forms. Sodium deoxycholate shows an intriguing variety of hydrates ranging from dihydrate to tetraand even octahydrate. ${ }^{[19]}$ The monographed creatine phosphate disodium salt exists in four interconverting hydrates ranging from 1.5 to 7 molar equivalents of water. ${ }^{[20]} \mathrm{A}$ comparably high hydration has been shown for cromolyn sodium, another disodium salt, which forms a non-stoichiometric hydrate incorporating between 5 and 8 molar equivalents of water. ${ }^{[21]}$ The trend of high water inclusion in disodium salts is further corroborated by pemetrexed disodium heptahydrate ${ }^{[22]}$ and disodium pamidronate pentahydrate, both monographed as these hydrates in the European Pharmacopoeia. Nedocromil disodium can exist in a heptahemi hydrate (7.5 molar equivalents of water per drug compound), a trihydrate, a monohydrate and an amorphous form that can contain between 1.5 and 3 molar equivalents of water. ${ }^{[23]}$

Due to the lower number of coordination sites, monosodium salts show lower hydration levels. They reveal, however, a seemingly equal potential for complexity of hydrated crystal forms Cefazolin sodium, as an example, is reported to form a pentahydrate, which is well characterized. ${ }^{[24]}$ However, there are hints towards additional sesqui- and monohydrated forms. Diclofenac sodium exists in a pentahydrate, two polymorphic tetrahydrates and a trihydrate. ${ }^{[25]}$ Naproxen sodium, finally, crystallises in a tetrahydrate, two polymorphic dihydrates and a monohydrate. ${ }^{[26]}$

Considering the problems that can be encountered with hydrate formation of API sodium salts, it is surprising that for the majority of the publications describing the crystal structure of the predominant phase of these compounds, crystallisation procedures in the case of patent literature, and solubility and

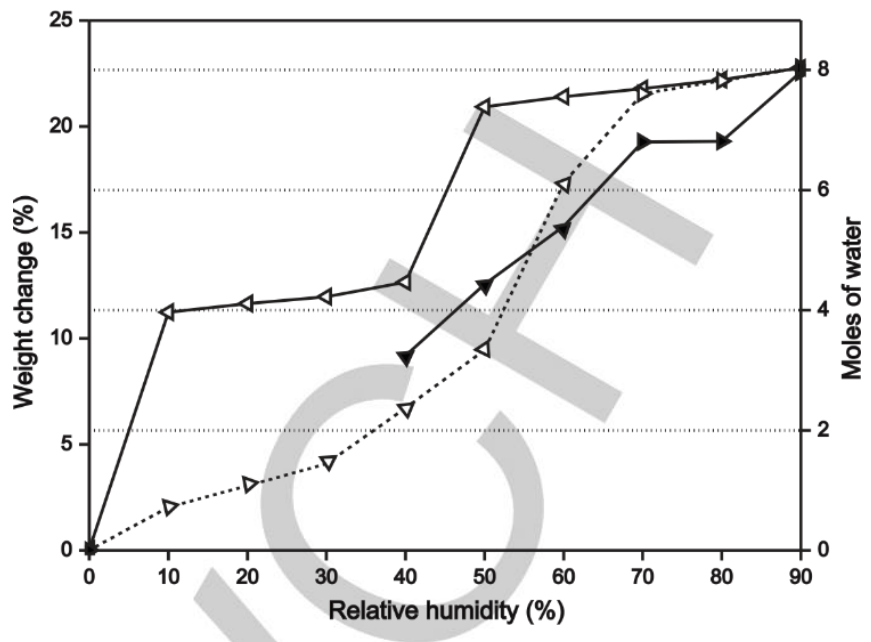

Figure 2. Sorption/desorption isotherms of the commercial sample of DTS Starting point is at $40 \%$ relative humidity $(\mathrm{RH})$ following a sorption step, then desorption to $0 \% \mathrm{RH}$, and finally a sorption up to $90 \% \mathrm{RH}$ again.

dissolution rate have been published. However, many of the pharmaceutical sodium salts have not been fully characterized, or their characterisation not published, and thus their phase transition behaviour cannot inform processing and manufacturing steps.

Diatrizoic acid is a radiocontrast agent that is monographed in the European ${ }^{[18]}$ and the United States Pharmacopoeia ${ }^{[27]}$ either as free acid or its meglumine or sodium salt. Whilst we have reported the solid-state characteristics of the free acid in detail, ${ }^{[28]}$ with a wide variety of hydrates, anhydrous crystal forms and solvates, and the disodium salt ethanol solvate, ${ }^{[29]}$ the sodium salt has not yet been investigated in depth. The presence of a tetrahydrate of the salt is reported by Tønnesen et al. ${ }^{[30]}$ whilst we have found this form to incorporate 4.5 molar equivalents of water per salt unit. ${ }^{[31]}$ The $X$-ray and neutron crystal structures show that most water is coordinated to the sodium cations, but there are channels in the structure through which the water can potentially freely diffuse in and out. Due to this interesting structure and the surprising supramolecular interactions in the free acid, we now report the solid-state behaviour of diatrizoic acid monosodium salt.

\section{Results and Discussion}

\section{The crystal forms}

This study showed that DTS can exist in at least five different hydrated crystal forms ranging from 0.3 to 8 molar equivalents of water per molecular unit. Powder X-ray diffraction shows all of them to be distinctly different crystal forms (Fig. 1). Only three of these (Hy8, Hy1 and $\mathrm{Hy}_{0.5-3.5}$ ) crystallise directly from solution, whilst the other two result from conversion of another form induced either by humidity or heating. In addition, a methanol trisolvate and a mixed $\mathrm{DMSO} / \mathrm{H}_{2} \mathrm{O}$ monosolvate directly crystallise from the respective solvents. An unsolvated crystal form could not be identified. 


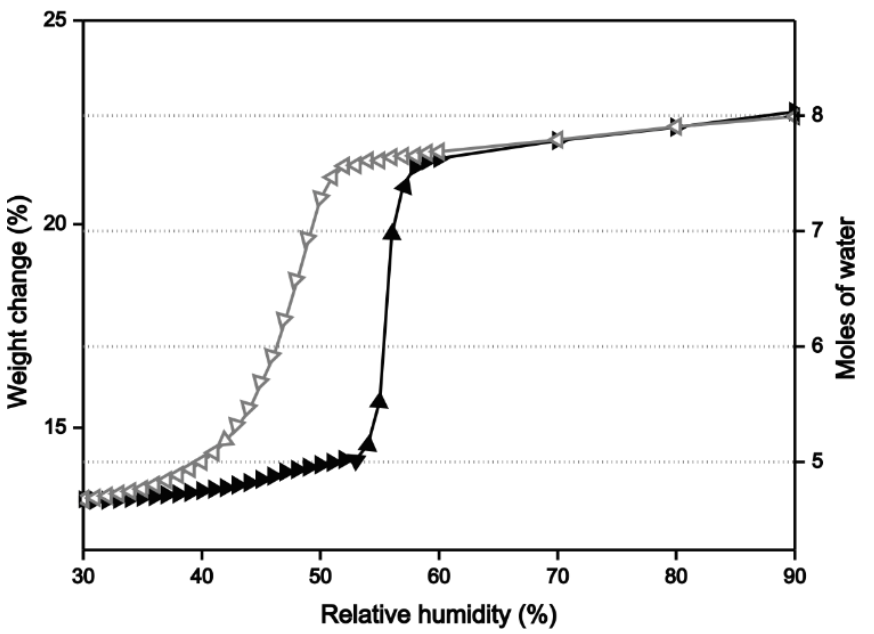

Figure 3. High resolution dynamic vapour sorption (DVS) isotherm of the transition between Hy8 and Hy4. The grey line represents desorption curve, the black line sorption.

\section{The hydrates}

The dynamic vapour sorption (DVS) isotherm of the amorphous commercial sample shows that the sample takes up a maximum of $22.76 \%$ of its weight over the range from 0 to $90 \%$ relative humidity (Fig. 2). At the same time, the amorphous material undergoes crystallisation (see ESI Figure S1). The weight gain corresponds well to a stoichiometry of 8 molar equivalent of water per DTS unit (calculated weight change 22.67\%), thus representing an octahydrate (Hy8). As described, it is very unusual for a mono-sodium salt to be this highly hydrated. The octahydrate can also be obtained by slurry conversion of the commercial form in water, which results in the formation of thin plates. The powder X-ray diffractogram of these plates is considerably different to the known tetrahydrate $(\mathrm{Hy} 4)$, even though the platy habit leads to preferred orientation (Fig. 1). However, most of the observed peaks are different to the calculated pattern of Hy4 based on the neutron structure. [28b]. Thermogravimetric (TG) analysis of Hy8 shows a large dehydration step with the weight loss of $37.7 \%$ (Fig. S2). This weight loss is not purely dehydration, as the sample was prepared as paste with surplus mother liquor to ensure full hydration of the octahydrate. The dehydration up to $150{ }^{\circ} \mathrm{C}$ (Fig. S3) reveals a two-step desolvation profile, even though no intermediate equilibrium is reached. Weight loss starts immediately with a slope of $-0.3 \%$ weight change per ${ }^{\circ} \mathrm{C}$ in the range from $22.6^{\circ} \mathrm{C}$ to $63.9^{\circ} \mathrm{C}$. The weight loss between these temperatures sums up to $12.81 \%$ and can be attributed to the evaporation of the mother liquor and surface-bound solvent. After this first weight loss, the slope becomes steeper $\left(-0.5 \%\right.$ per $\left.{ }^{\circ} \mathrm{C}\right)$ between $63.9{ }^{\circ} \mathrm{C}$ to $110.1{ }^{\circ} \mathrm{C}$. The second weight loss equals $22.56 \%$, which corresponds to 7.963 molar equivalents of water and thus the desolvation of intact Hy8. This two-step profile was confirmed with differential scanning calorimetry (DSC), which shows two overlapping endothermic events corresponding to the events observed in the TG trace (Fig. S3).

Desorption of $\mathrm{Hy} 8$ by reducing the humidity leads to the formation of the tetrahydrate Hy 4 below $50 \% \mathrm{RH}$ (Fig. 2), as was confirmed by powder X-ray diffraction (PXRD, Fig. 1). Even

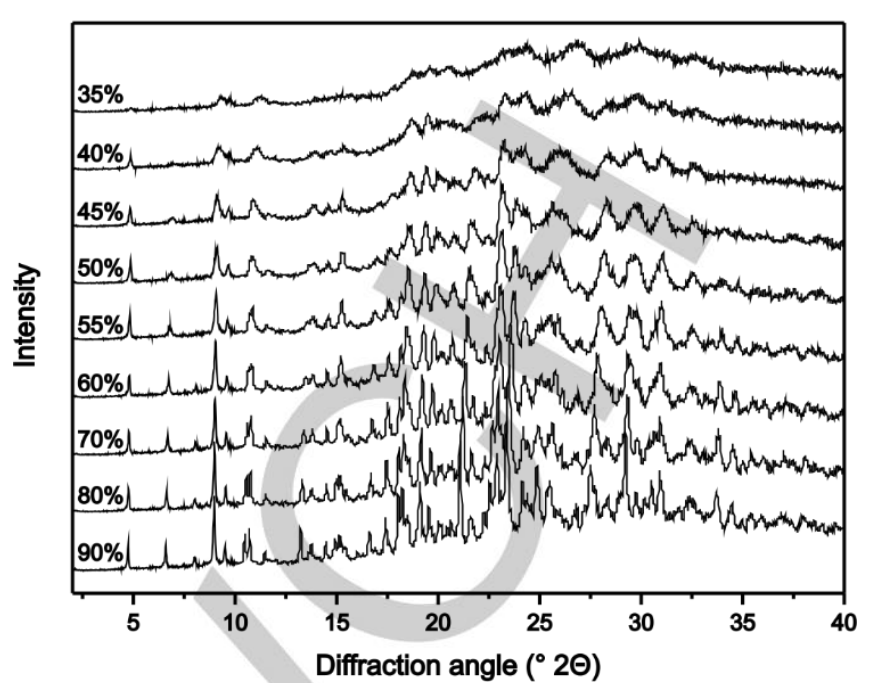

Figure 4. Humidity controlled PXRD patterns starting from $\mathrm{Hy} 8$ at $90 \% \mathrm{RH}$ and passing through the transition to $\mathrm{Hy} 4$.

though the powder pattern of this form show resemblance to the pattern calculated from the single crystal structure we have published earlier, this crystal form suffers from pronounced preferred orientation and the presence of a phase transition between the temperature of the powder and the single crystal measurements ( $300 \mathrm{~K}$ vs. $120 \mathrm{~K}$ ) cannot be unambiguously ruled out.

Hy 4 could also be obtained by storing the commercial sample in aqueous slurry and drying the powder at room conditions. This hydrate cannot be obtained by direct crystallisation but only by dehydration of Hy8. Thus, its habit resembles that of Hy8 (Fig. S4). Surprisingly, the crystals of Hy4 are perfectly translucent and do not show any signs of pseudomorphosis, indicating the close structural relationship of these two highly hydrated crystal forms, and a single-crystal-to-single-crystal transition. High-resolution DVS shows the reversibility of the transition between $\mathrm{Hy} 8$ and $\mathrm{Hy} 4$ (Fig. 3). Above $50 \% \mathrm{RH}, \mathrm{Hy} 8$ is stable but releases almost 0.5 molar equivalents of its crystal water, which points towards the presence of channels within the crystal structure, from which the water can diffuse comparably freely depending on the environmental water pressure, i.e. the relative humidity. Below $50 \% \mathrm{RH}$, the sample releases a further 2.3 molar equivalents of water in a steep step. At $43 \% \mathrm{RH}$, the abrupt weight loss levels off but a further 0.4 molar equivalents of water is released until reaching $30 \% \mathrm{RH}$. The final water content of $\mathrm{Hy} 4$ is verified as 4.5 molar equivalents at $30 \% \mathrm{RH}$ by DVS. This continuous water release is characteristic for non-stoichiometric hydrates, for which the water content solely depends on the water pressure of the surrounding atmosphere. ${ }^{[7]}$ The non-stoichiometric character of the phase transition is corroborated by humidity-controlled PXRD (Fig.4). The peak positions of the initial octahydrate shift to the higher diffraction angles and thus smaller $d$ spacing. There is no abrupt change between peak positions or peaks growing into the patterns, which would indicate a stoichiometric transition to a novel crystal form. It is clearly visible that the peaks become broader and less intense at lower $\mathrm{RH}$ levels, which indicates loss of crystallinity during the water release. The lower crystallinity also explains the hysteresis observed in the hydration of $\mathrm{Hy} 4$ to $\mathrm{Hy} 8$. 


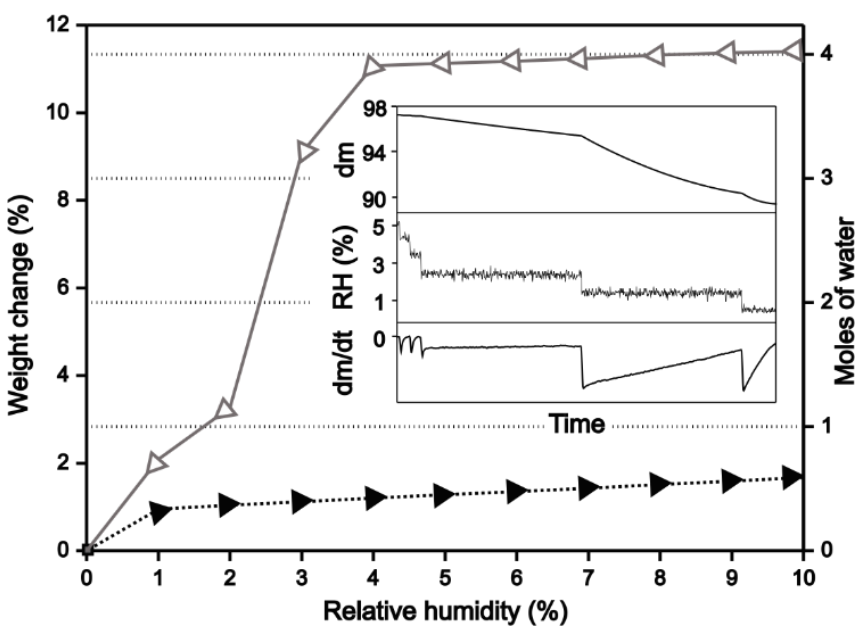

Figure 5. High resolution DVS of the dehydration of Hy4. Insert represents the mass change $(d m)$, respective $\mathrm{RH}$ levels and mass change rate $(d m / d t)$ of the steps below $5 \% \mathrm{RH}$.

Generally, the presence of a hysteresis would point towards a stoichiometric hydrate, for which the hydration and dehydration steps are kinetically controlled. Non-stoichiometric hydrates do not show the kinetic delay, as the water from the environment can freely diffuse in and out of the features of the crystal structure, and the sorption and desorption isotherms follow the exact same trace. ${ }^{[32]}$ However, the partial loss of crystallinity of Hy4 after the transition introduces a kinetic element, i.e. recrystallization, into the hydration, which leads to this event being delayed to $55 \% \mathrm{RH}$, above which a rapid water uptake leads to Hy8.

Storing Hy 4 between 40 and $10 \% \mathrm{RH}$ leads to only a slight variation in water content between 4.5 and 4 molar equivalents of water. It is thus likely that the structure of Tønnesen et al. ${ }^{[30]}$ has been measured at the lower end of this humidity spectrum and therefore resulted in four water molecules being refined. Below $10 \% \mathrm{RH}$, the remaining water is released (Fig. 2). High-resolution DVS of this transition (Fig. 5) shows that a step-like dehydration takes place below $4 \% \mathrm{RH}$, in which the sample loses $7.8 \%$ of its weight (2.76 molar equivalents of water). This mass loss leads to an intermediate monohydrate, which is stable at $2 \% \mathrm{RH}$ but undergoes a further mass loss below this humidity. Unfortunately, this transition could not be followed by humidity-controlled PXRD, as the sample shows too low crystallinity to make out distinct peaks (Fig. S5). It is not clear whether the sample completely amorphises or is of too low crystallinity and appears X-ray amorphous in this experiment. From the DVS data, however, it is conclusive that this intermediate is indeed a crystalline phase, as the mass of the sample is in equilibrium at $2 \% \mathrm{RH}$, whilst this is not reached at $1 \% \mathrm{RH}$ (Fig. 5 insert). At the lower humidity, the sample is in the process of releasing the remaining incorporated water; however, the mass step is not at its final value, as clearly shown by the remaining slope in the $d \mathrm{~m} / d t$ curve. Even though only existing at an extremely narrow humidity range, the monohydrate does not represent a kinetic intermediate, as the crystal form is clearly stable over six hours (potentially longer) under these conditions, which is the time that this step has taken in the measurement.

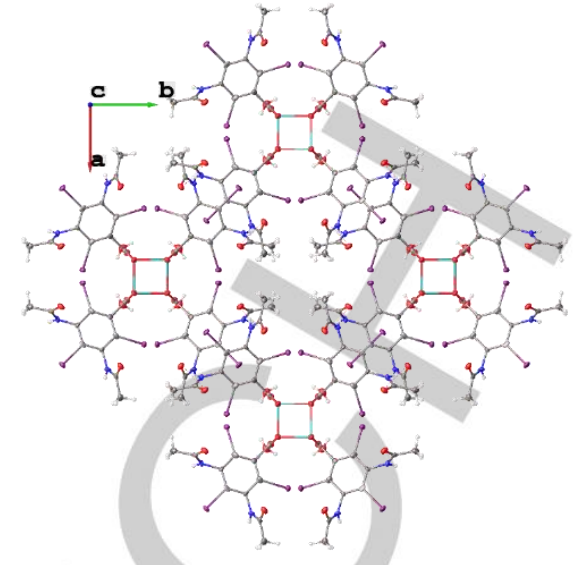

Figure 6 Packing representation of the single crystal structure of Hy1. For this and all following structural representations, anisotropic displacement parameters are drawn at $50 \%$ probability, and the colour code is carbon grey, nitrogen blue, oxygen red, iodine purple, sodium cyan and hydrogen light grey.

The sorption of the completely dehydrated sample leads to a further hydrated form, which contains between 0.3 and 1 molar equivalents of water $\left(\mathrm{Hy}_{0.3-1}\right)$ and takes up this amount in a very linear manner between 1 and $40 \% \mathrm{RH}$ (Fig. 5 and S6). It is not conclusive from the data, especially considering the low crystallinity of the sample and thus lack of clear humiditycontrolled PXRD patterns, whether this hydrate is stoichiometric or non-stoichiometric in nature.

The hydrate $\mathrm{Hy}_{0.3-1}$ obtained in the DVS could be reproduced by two different experimental routes. Firstly, a sample of the octahydrate was dried at $25^{\circ} \mathrm{C}$ in dry nitrogen flow over ten hours using TG to directly mimic the conditions of the DVS. Secondly, Hy8 was dehydrated by $\mathrm{TG}$ at $150{ }^{\circ} \mathrm{C}$ for ten minutes. The resulting materials show the same PXRD patterns (Fig. 1), and even though the peaks have low intensity, their positions are distinctly different to those of Hy8 and Hy4. Both samples showed a comparable weight loss of approximately $1 \%$ corresponding to 0.4 molar equivalents of water after being exposed to environmental humidity.

Upon sorption above $40 \% \mathrm{RH}, \mathrm{Hy}_{0.3-1}$ takes up water in a slow manner and transforms directly into Hy8. The slow transition is reminiscent of the transition of $\mathrm{Hy} 4$ to $\mathrm{Hy} 8$ and presumably hampered by the low crystallinity of the sample and potential amorphous fraction introducing a kinetic component to the event. This conclusion is further corroborated by humidity-controlled PXRD (Fig. S7), which shows a slow transition from the X-ray amorphous sample to higher crystallinity at higher humidity, i.e. when the sample transforms into Hy8.

To obtain the intermediate hydrated forms through another pathway, a full crystallisation screening was conducted. The predominantly crystallising form is another hydrate, which forms needle-shaped crystals (Fig. S4). TG analysis shows $3.26 \%$ weight loss (Fig. S2), which corresponds to 1.1 molar equivalents of water thus classifying the novel crystal form as a monohydrate (Hy1). PXRD analysis shows clearly different peak positions to the other hydrated forms (Fig. 1). Single crystals of Hy1 grown in slowly cooled ethanol solution crystallise in the tetragonal space group $14 \mathrm{~cm}$ with half a molecular unit in the asymmetric unit. The packing shows both linear coordination polymeric strands as well 


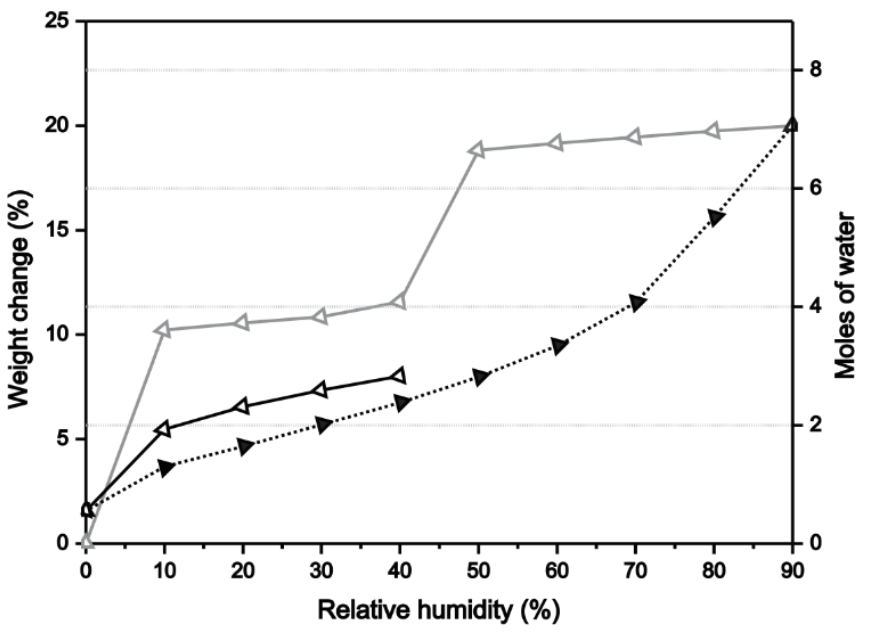

Figure 7 DVS isotherm of Hy1 starting form room conditions down. Isotherm is normalised to lowest point of the measurement.

as hydrogen bonding motifs running on parallel along the unique $c$ axis (Fig. 6). The sodium cations coordinate to the deprotonated acid, as is also the case for Hy4, whilst the coordination scheme between the two crystal forms is different. In Hy4, the carboxylate coordinates to the sodium ion through one oxygen atom, whilst the other accepts a hydrogen bond from a water molecule. In Hy1, one carboxylic oxygen atom likewise coordinates to one sodium cation, but the other instead coordinates to two more $\mathrm{Na}$ cations in the next layer along the $c$ axis. This motif results in a square of alternating sodium and oxygen atoms, in which each carboxylic acid bonds to three sodium cations and each sodium bonds to three carboxylates. A fourth coordination bond of the sodium ion is realised by bonding to the water molecule. Surprisingly, the incorporated water molecule does not donate any hydrogen bonds to the surrounding molecules and thus acts merely as filler for the coordination shell and space filler for the structure. The diatrizoate anions show the cis conformation in which the amide carbonyl moieties point towards one side of the central ring and the amino moieties are directed to the opposite side. ${ }^{[28 \mathrm{~b}]}$ This enables catemeric hydrogen bonding between the coordination polymeric strands along the $c$ axis. No halogen bonds are realised in this structure. Overall, the packing of $\mathrm{Hy} 1$ is more efficient with a calculated density of $2.50 \mathrm{vs} .2 .33 \mathrm{~g} \mathrm{~cm}^{-3}$ for Hy4, which points towards a lower energy of the monohydrate. This is reflected in the dominance of $\mathrm{Hy} 1$ as the crystallisation result of most solution crystallisations (Table S1). The DVS isotherm of Hy1 is distinctly different to that of the transient monohydrate obtained from dehydration of Hy4 (Fig. 7). It is noteworthy that even though the water content of the directly crystallised $\mathrm{Hy} 1$ as determined by Karl-Fischer titration is slightly higher than 1 mole of water $(3.75 \%$ corresponding to 1.375 molar equivalents of water), the same sample submitted to DVS measurements contains $8 \%$ of volatile inclusion. This could be due to a low impurity of the material, potentially a higher hydrate, even though no impurities were detected by PXRD (Fig. 1). It is also possible that this hydrate shows non-stoichiometric characteristics and can contain a higher amount of water at higher humidity. The DVS isotherm starting from $40 \% \mathrm{RH}$ (room conditions) towards lower humidity shows a gradual release of the incorporated solvent with an increase in

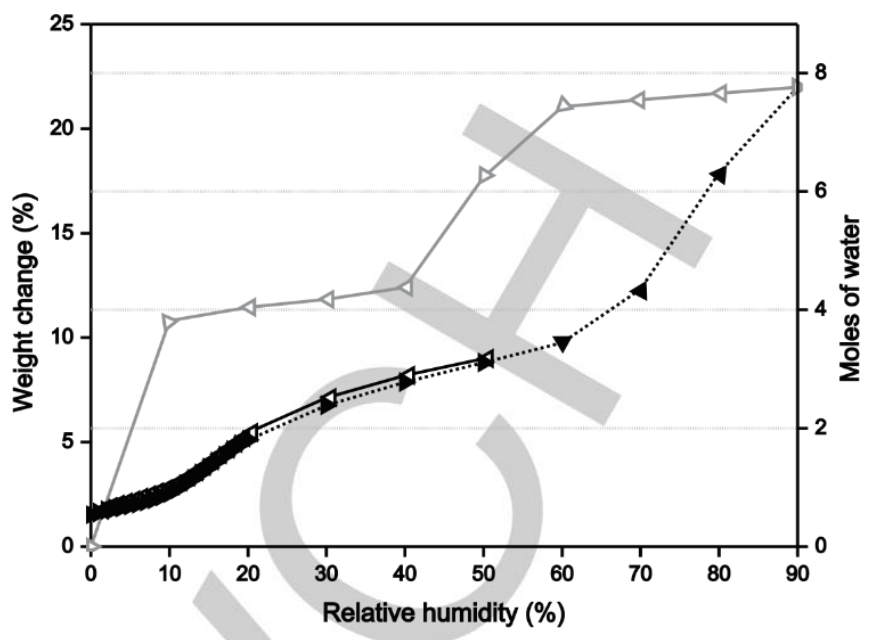

Figure 8 DVS isotherm of $\mathrm{Hy}$ 2.4-3.4 starting form room conditions down. Isotherm is normalised to lowest point of the measurement.

rate towards $0 \% \mathrm{RH}$. The sorption isotherm does not follow the same path as the desorption, but runs in parallel at a lower weight. Since the atmosphere in the instrument only contains water vapour, it can be safely assumed that this increase in weight corresponds to the take-up of water. This would corroborate the assumption of a low fraction impurity containing either a larger amount of water or a heavier solvent, e.g. ethanol from the solution crystallisation. The sample shows an almost linear uptake of water over the range from $10 \%$ to $60 \% \mathrm{RH}$, which corroborates the hypothesis of this hydrate being nonstoichiometric. Unfortunately, the sample amount of $\mathrm{Hy} 1$ accessible by crystallisation is too low to perform humidity controlled PXRD measurements, proving this hypothesis. At humidity levels between $60 \%$ and $90 \% \mathrm{RH}$, the weight gain rate increases whilst the sample converts to Hy8. This is clearly shown by the following desorption curve, which is identical to that of the octahydrate.

A second hydrated crystal form could be obtained by direct crystallisation. The plate-shaped crystals grow concomitantly with Hy1 from ethanol solution, and are distinctly different by PXRD (Fig. 1). TG measurements reveal a water content of $6.83 \%$, which correlates with 2.6 molar equivalents of water.

The DVS isotherm of this hydrate reveals that the sample contains $8.99 \%$ volatile component (3.2 molar equivalents of water), again at a slightly higher weight percentage when normalised to the lowest point of the isotherm being zero (Fig. 8). Upon lowering the humidity, the sample loses weight in a continuous manner, which speeds up between 20 and $10 \% \mathrm{RH}$ whilst stabilising again between 10 and $0 \% \mathrm{RH}$. The lowest water content of this hydrate is 0.5 molar equivalents. The sorption isotherm follows an almost identical path to the desorption proving the non-stoichiometric character of this hydrate. The highest water content of this form is reached with $9.75 \%$ (3.44 molar equivalents of water) at $60 \% \mathrm{RH}$, thus the ranges of this hydrate are between 0.5 and 3.5 molar equivalents of water $\left(\mathrm{Hy}_{0.5-3.5}\right)$. Above $60 \% \mathrm{RH}$, the sample takes up water at a higher rate and transforms to the octahydrate. Almost full hydration of Hy8 is reached indicating only minor traces of impurities. The second desorption isotherm traces exactly that of Hy8. 


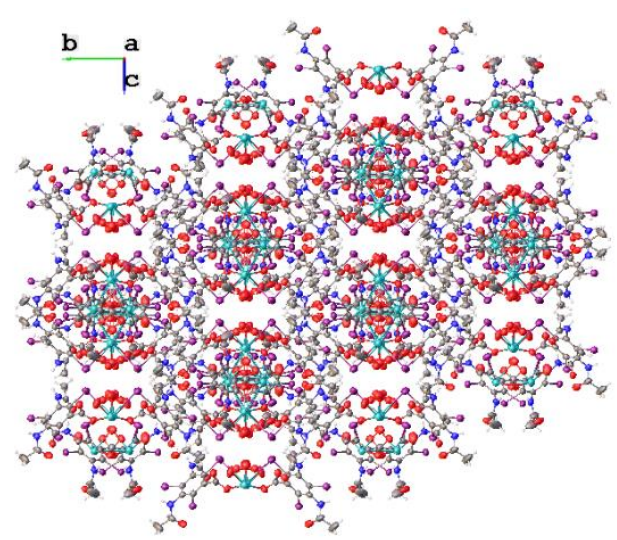

Figure 9 Packing of the crystal structure of $\mathrm{Hy}_{0.5-3.5}$ along the crystallographic a axis with clearly visible pores.

Due to the small size of the individual crystals, diffraction data of $\mathrm{Hy}_{0.5-3.5}$ were collected on the synchrotron beamline $\mathrm{I19}$ at Diamond Light Source, Oxfordshire, UK. The structure solution shows that this hydrate crystallises in the tetragonal space group $14_{1} / a$ with one molecular unit in the asymmetric unit. Surprisingly, only 1.6 molar equivalents of water can be refined, even though additional electron density points towards more disordered water in this structure. As in the other hydrates, the carboxylate moieties coordinate to the sodium cations; however, in $\mathrm{Hy}_{0.5-3.5}$ only one of the oxygen atoms is involved in the coordination. The resulting motif is a slightly twisted cube with alternating sodium and oxygen atoms at its corners (Fig. S8). Each sodium cation furthermore coordinates to a water oxygen atom, whilst two half-occupied water positions bridge two faces of the cube. Comparable to Hy4, the second oxygen atom of the carboxylate moiety is involved in hydrogen bonding with a coordinate water molecule (donoracceptor heavy atom distance: $2.74(2) \AA$ A). The hydrogen bonding motif found between the diatrizoate ions resembles that of $\mathrm{Hy} 4$ : centrosymmetric dimers show hydrogen bonding between one amide $\mathrm{N}-\mathrm{H}$ moiety to the non-coordinated oxygen atom of the carboxylate, whilst the $\mathrm{N}-\mathrm{H}$ of the second amide hydrogen bonds to a third diatrizoate amide carbonyl. The latter hydrogen bond leads to spirals along the $4_{1}$ axis. Halogen bonds involving the iodine atoms are realised with both amide carbonyl oxygens as well as to the disordered water.

The structure contains a 2-dimensional network of solventaccessible channels, which run along the crystallographic $a$ and $b$ axes (Fig. 9). These channels are the reason for the nonstoichiometric behaviour observed by DVS, as water from the environment can diffuse in and out of the structure without changing the overall scaffold. This hypothesis is corroborated by the remaining electron density, which is located within the channels and points towards additional disordered water molecules. Compared to $\mathrm{Hy} 4$ and $\mathrm{Hy} 1, \mathrm{Hy}_{0.5-3.5}$ has with $2.14 \mathrm{~g} \mathrm{~cm}^{-3}$ the lowest density, which may be due to missing modelled water. However, the low density points towards the highest energy and thus the lowest stability of this crystal form. This is corroborated by the occurrence of $\mathrm{Hy}_{0.5-3.5}$ in only one crystallisation experiment and even then only concomitantly with the more stable Hy1.

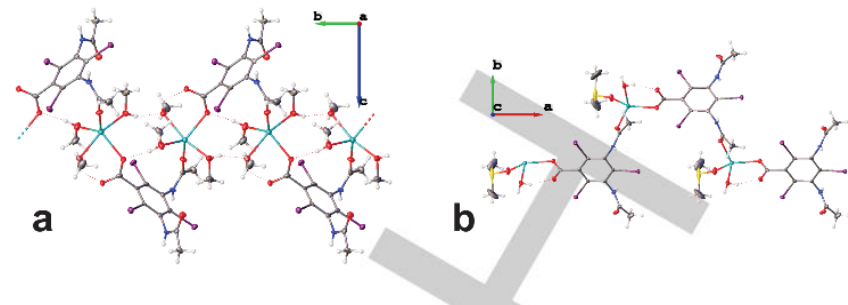

Figure $101 \mathrm{D}$ coordination polymers of the (a) methanol and (b) DMSO/ $\mathrm{H}_{2} \mathrm{O}$ solvates of DTS.

\section{The solvates}

In addition to the hydrated crystal forms, two solvates of DTS were found to crystallise from methanol and DMSO, respectively (Fig. S9). The methanol solvate shows a weight loss of $13.03 \%$ by TG, which corresponds to 3 molar equivalents of methanol (calculated weight loss $13.113 \%$, Fig. S10). It is not surprising that such a high number of methanol molecules are incorporated by DTS, especially considering the complex hydration behaviour as discussed above, as methanol is only slightly bigger than water and can coordinate to the sodium cation in a comparable manner. The weight loss of the methanol solvate starts already at room temperature indicating the low stability of this solvate, and it is likely that desolvation occurs under dry conditions. The low stability is additionally reflected by the crystallisation of Hy1 from methanol by evaporation under ambient conditions (approximately $40 \% \mathrm{RH}$ ). Single crystals of the methanol solvate suitable for X-ray diffraction grew in a slowly cooled methanol solution. The structure crystallises in the monoclinic space group $P 2_{1} / C$ with one molecular unit in the asymmetric unit. Three solvent molecules coordinate to the sodium cation, proving this structure to be a trisolvate. In addition, the carboxylate group coordinates to a sodium ion, as well as one amide carbonyl. The latter interaction is not realised in any of the hydrate structures and leads to 1D coordination polymeric chains along the crystallographic $b$ axis (Fig. 10a). The second amide group accepts a hydrogen-bond from the amide $\mathrm{N}-\mathrm{H}$ moiety of a neighbouring anion, which connects the polymeric chains with each other. Two of the methanol molecules are hydrogen-bonding to the carboxylate moiety of the anion, whilst the third methanol donates a hydrogen bond to a neighbouring methanol molecule. There are no clear channels for the solvent to diffuse through and its hydrogen-bonded nature in this structure does not explain the relatively low stability of this solvate.

Precipitation of DTS from DMSO solution with antisolvent yields a metastable solvate containing one molecule of DMSO and water each per DTS, which is confirmed by the experimental weight loss by TG (Fig S10). This mixed solvate crystallises in the orthorhombic space group $P c a 2_{1}$ with one molecular unit in the asymmetric unit. The sodium cation has the lowest coordination number with only four oxygen atoms (one from each solvent, two from diatrizoate) interacting with it. The coordination of two oxygen atoms of the anion through the carboxylate as well as one amide carbonyl leads to the presence of 1D coordination polymeric chains comparable to the methanol solvate (Fig. 10b). However, the geometry of the chain in this solvate is more open and linear due to the lack of hydrogen bonds, which in the case of the methanol solvate leads to a spiral with a short pitch length. 


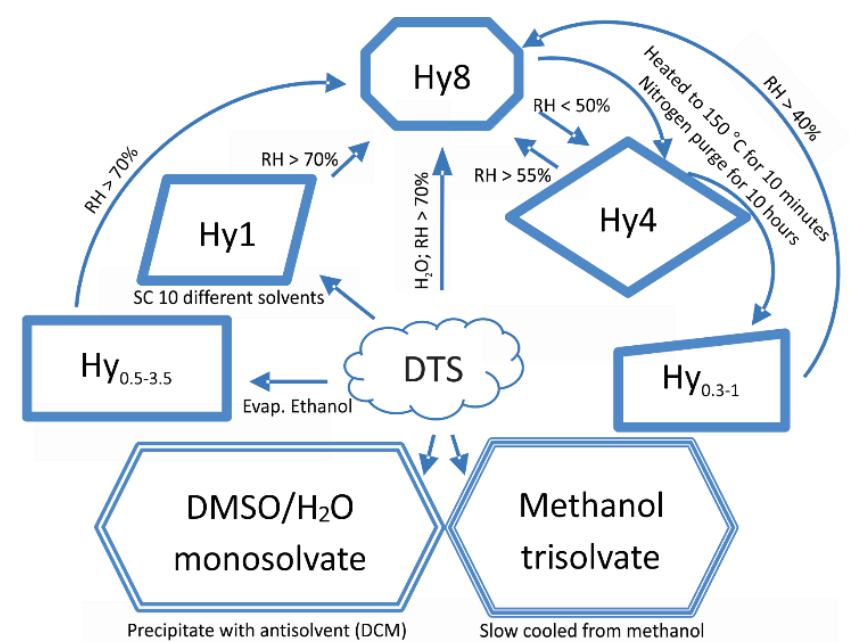

Figure 11 Schematic representation of the crystal forms and transformation pathways of DTS.

The open chains of the DMSO/ $\mathrm{H}_{2} \mathrm{O} 1: 1$ solvate pack closely on top of each other leading to a herringbone packing. Whilst the incorporated water is donating hydrogen bonds to both the carboxylate and the amide moiety of the anion, the DMSO molecules are only coordinated to the sodium ion through their oxygen atoms and thus show higher motion around this hinge (detected as larger ADPs, Fig. S11). Surprisingly, though, the two different solvent species leave the crystal lattice at the same time, as observed by TG, showing that the initial crystal structure is not stable without either of the guest components and collapses. Even though this mixed solvate is metastable, it gives another indication of the high attraction of DTS to water.

\section{Conclusions}

Diatrizoic acid monosodium salt exists in at least five hydrated crystal forms and two solvates. The hydrates range in water content from 0.3 to 8 molar equivalents (Fig. 11). Three of them (Hy8, $\mathrm{Hy} 1$ and $\mathrm{Hy}_{0.5-3.5}$ ) crystallise directly from solution whilst the remaining hydrates ( $\mathrm{Hy} 4$ and $\mathrm{Hy}_{0.3-1}$ ) are obtained by dehydration of Hy8 only. At high humidity levels, all hydrates convert to Hy8. The solvates contain three moles of methanol or one mole each of DMSO and water in a mixed solvate. Structural analysis of three hydrates and both solvates show a strong involvement of the sodium cation but additional stabilising hydrogen bonding. The monohydrate and both solvates show 1D coordination polymers through interaction between the organic anion and the sodium ion. Except for Hy4, all incorporated water is directly coordinated to the cation and hydrogen bonds to other moieties of the crystal structure. No anhydrous crystal form of DTS could be identified.

This intriguing pharmaceutical salt exemplifies the importance of in-depth data on the solid-state behaviour of a sodium salt under variable humidity conditions. It is obvious that the incorporation of water into the crystal structures stabilises them, as apart from the methanol solvate, no water-free modification could be obtained. The interchanging characteristics of the hydrates additionally highlight the importance of humidity- controlled experiments to fully understand pharmaceutical sodium salts before formulation and manufacturing. DTS can contain between 0.3 and 8 molar equivalents of water depending on the crystal form and relative humidity, which will have an impact on dose, solubility and uniformity of any produced solid formulation. The frequent phase changes upon varying the humidity, as well as potential amorphisation upon release of all incorporated water, presents an additional complication for manufacturing and stability of the final formulation. Even though DTS is not currently marketed as a solid, it is a good model compound to show up the potential hazards of sodium salts in water-containing environments and advocates for full and in-depth characterisation of other sodium salts used pharmaceutically.

\section{Experimental Section}

\section{Materials}

Sodium diatrizoate $(\geq 98.0 \%)$ (DTS) was purchased from Sigma-Aldrich and used unrefined. Acetone $(\geq 99.8 \%)$, acetonitrile $(\geq 99.9 \%)$, n-amyl alcohol $(\geq 99.0 \%)$, 1-butanol $(\geq 99.4 \%)$, 2-butanol $(\geq 99.5 \%)$, dimethyl sulfoxide (DMSO) ( $\geq 99.9 \%), \quad 1,4$-dioxane $(\geq 99.0 \%), \quad N, N$-dimethyl formamide (anhydrous $99.8 \%$ ), dichloromethane $(\geq 99.8 \%)$, ethyl acetate (anhydrous $\geq 99.8 \%)$, 2-butanone (ethyl methyl ketone) $(\geq 99.0 \%)$, hexane (anhydrous 95.0\%), methanol ( $\geq 99.6 \%$ ), 1-propanol (anhydrous 99.5\%), 2-propanol (anhydrous 99.5\%), tetrahydrofuran (anhydrous 99.9\%), were purchased from Sigma-Aldrich, diethyl ether (99.99\%) and chloroform $(99.98 \%)$ were purchased from Fisher chemicals, ethanol $(\geq 99.8 \%)$ was purchased from Fluka, while nitromethane (96.0\%) was purchased from Acros organics. All solvents were used without further purification. The Karl Fisher titration reagent used was HYDRANAL®- Coulomat AG (SigmaAldrich Laborchemikalien $\mathrm{GmbH}$, Seelze, Germany)

\section{Methods}

Crystallisation: Slow and fast cooling, evaporation and precipitation crystallisation methods were used. Approximately $20 \mathrm{mg}$ of DTS was used in each of the crystallisation experiments. For the cooling experiments, DTS was dissolved in sufficient amounts of each of the 21 solvents to produce a hot saturated solution. This was then cooled at different rates; slow cooled samples were left to cool to room temperature in an insulating wooden block while fast cooled samples were quenched in an ice and water bath. For evaporation, the material was dissolved in sufficient solvent to produce an undersaturated solution and left to evaporate at ambient conditions. Precipitation was conducted by producing a hot saturated solution and adding dichloromethane or diethyl ether as antisolvents, depending on miscibility. In the case of the fast cooling and precipitation experiments, the crystals were immediately removed from solution once they formed, whereas evaporation and slow cooling experiments were left for 24 hours before analysis.

Thermogravimetric analysis scans were carried out with a Q 500 (TA Instruments, New Castle, DE, USA). Nitrogen served as the purge gas at a flow rate of $60 \mathrm{~mL} \mathrm{~min}^{-1}$. Samples of 1 to $5 \mathrm{mg}$ were accurately weighed $( \pm 0.01 \mathrm{mg})$ into $50 \mu \mathrm{L}$ platinum pans. A heating rate of $10^{\circ} \mathrm{C} \mathrm{min}{ }^{-1}$ was used and each sample was heated from $25^{\circ} \mathrm{C}$ to $300^{\circ} \mathrm{C}$.

Differential scanning calorimetry scans were carried out on Q 2000 (TA Instruments, New Castle, DE, USA). Nitrogen served as the purge gas at a flow rate of $60 \mathrm{~mL} \mathrm{~min}-1$. Approximately 1 to $5 \mathrm{mg}$ of the sample were accurately weighed $( \pm 0.01 \mathrm{mg})$ using a microbalance (Sartorius, Göttingen, Germany), loaded into aluminium standard pans (TA Instruments, New 
Castle, DE, USA) and sealed. A heating rate of $10{ }^{\circ} \mathrm{C} \mathrm{min}{ }^{-1}$ was used and each sample was heated from $25^{\circ} \mathrm{C}$ to $300^{\circ} \mathrm{C}$.

Karl Fischer titrations for water content determination were performed using a Metrohm AG 899 coulometer titrator (Metrohm Ltd., Herisau, Switzerland). The medium was titrated to dryness before each analysis. Between 5 and $10 \mathrm{mg}$ of sample was weighed $( \pm 0.1 \mathrm{mg})$ using a Kern ABJ 200-4NM analytical balance (Kern \& Sohn GmbH, Balingen, Germany) for each analysis

Gravimetric moisture sorption analysis was carried out using a humidity and temperature controlled dynamic vapour sorption (DVS) apparatus, (Surface Measurement Systems, Boro Labs Ltd. Aldermaston, Berks, U.K.) equipped with Cahn D200 digital recording ultra-microbalance with a mass resolution of $\pm 0.01 \mu \mathrm{g}$. Initially, samples between 10 to $15 \mathrm{mg}$ were accurately weighed $( \pm 0.1 \mathrm{mg})$ using a Kern ABJ 200-4NM analytical balance (Kern \& Sohn GmbH, Balingen, Germany) and then loaded in TA Tzero pans (TA Instruments, Switzerland). Moisture uptake and release cycles (reported relative to the dry weight) were monitored over a sorption/desorption range of $0 \%$ to $90 \%$ relative humidity $(\mathrm{RH})$, with increments of $10 \% \mathrm{RH}$, at a constant temperature of $25{ }^{\circ} \mathrm{C}$. Higher resolution runs with $1 \% \mathrm{RH}$ step intervals were performed at $\mathrm{RH}$ at phase transitions points were observed. The equilibrium condition for each step was set to a mass constancy of $\pm 0.01 \%$.

Fourier transform infra-red spectroscopy (FTIR) spectra of solid phases were collected on a Spectrum Two IR Spectrometers (PerkinElmer, Massachusetts, U.S.A). Samples were placed on a diamond Attenuated Total Reflectance (ATR) accessory. Four scans were collected for each sample at a resolution of $2 \mathrm{~cm}^{-1}$ over a wavenumber region of $4000 \mathrm{~cm}^{-1}$ to $600 \mathrm{~cm}^{-1}$.

Powder X-ray diffraction (PXRD) data were obtained using an Empyrean powder diffractometer (PANalytical, Almelo, Netherlands) equipped with $\mathrm{Cu}$-Ka radiation (wavelength of $1.541874 \AA$ ), graphite monochromator, 0.2 $\mathrm{mm}$ fixed Soller slits and PIXcel detector. The operating tube voltage of 40 $\mathrm{kV}$ and tube current of $40 \mathrm{mV}$ were used. For each sample, a scan was conducted in the Bragg-Brentano $(\theta-2 \theta)$ geometry between 2 to $40^{\circ} 2 \Theta$, at rate of $0.5^{\circ} 2 \Theta$ per minute. The samples were prepared as dry powder and placed on a zero-background sample holder. Humidity PXRD data were obtained using a D8 Discover powder diffractometer (Bruker, Coventry, UK) equipped with a Cu-Ka source (wavelength of $1.5406 \AA$ ), primary motorised slits set at $10 \mathrm{~mm}$ fixed sample size, Soller slits, secondary motorised slits set at $4^{\circ}$ opening angle and a LynxEye detector. The operating tube voltage of $40 \mathrm{kV}$ and tube current of $40 \mathrm{~mA}$ were used and the diffractometer was equipped with a $\mathrm{CHC}$ plus+ humidity chamber. The sample was mounted on a zero-background holder in the chamber, and all scans were conducted in the Bragg-Brentano (theta-2theta) geometry from 2 to $40^{\circ} 2 \Theta$, with a step size of $0.018^{\circ} 2 \Theta$ and a time per step of 0.2 seconds. The sample was held at $25^{\circ} \mathrm{C}$ and the humidity varied in steps, with the sample allowed to equilibrate for 2 hours at each step. For temperature-controlled PXRD run between humidity runs, humidity generation was switched off, and the temperature was varied in steps, with 2 hours equilibration time allowed at each step.

Single crystal X-ray diffraction data were obtained on crystals coated in perfluoro polyether oil and mounted on MiTeGen sample holders. The samples were placed directly into the pre-cooled cryostream. Datasets of $\mathrm{Hy} 1$ and the DMSO $\mathrm{H}_{2} \mathrm{O}$ solvate were collected on a D8 diffractometer (Bruker, Coventry, UK) operated with Mo-Ka radiation, double mirror monochromator and Photon detector. The methanol solvate was measured on an Xcalibur diffractometer (Oxford Diffraction, Oxford, UK) operated with graphite-monochromated Mo-Ka radiation. The dataset of Hy $0.5-3.5$ was collected on instrument I19 at the Diamond Light Source, Didcot, UK. The data were reduced using APEX2 software package (Bruker, Coventry, UK), solved with olex.solve and refined with olex.refine as included in the Olex2 software package. ${ }^{[33]}$ All non-hydrogen atoms were identified from electron density maps and refined anisotropically, whilst hydrogen atoms were modelled in geometrically ideal positions and refined as a riding model.

Hot-stage microscopy observations were carried out with a Leica DM 2700P polarising microscope (Leica Microsystems $\mathrm{GmbH}$, Wetzlar, Germany) equipped with THMS600 heating and freezing microscope stage and controller (Linkam Scientific Instruments, Tadworth, U.K.). The setup is completed with QICAM Fast1394 (Qimaging, British Colombia, Canada) colour video camera attachment, supported by Linksys32 version 2.4.3.

\section{Acknowledgements}

The authors thank Diamond Light Source for allocation of beamtime through the Durham-Newcastle network (MTMT 8682) and the instrument scientists for their kind support. MNMN thanks the Ministry of Higher Education Malaysia and the University of Teknologi MARA for funding.

Keywords: Pharmaceuticals • Crystal hydrate $•$ Crystal structure - sodium salt

[1] S. M. Berge, L. D. Bighley, D. C. Monkhouse, J. Pharm. Sci. 1977 66, 1-19.

[2] a R. J. Bastin, M. J. Bowker, B. J Slater, Org. Process Res. Dev. 2000, 4, 427-435; b P. L. Gould, Int. J. Pharm. 1986, 33, 201-217.

[3] L. Kumar, A. Amin, A. K. Bansal, Pharm. Technol. 2008, 3.

[4] a D. A. Haynes, W. Jones, W. D. S. Motherwell, J. Pharm. Sci. 2005, 94, 2111-2120; b G. S. Paulekuhn, J. B. Dressman, C. Saal, J. Med. Chem. 2007, 50,6665-6672; c A. T. M. Serajuddin, Adv. Drug Delivery Rev. 2007, 59, 603-616.

[5] K. R. Morris, M. G. Fakes, A. B. Thakur, A. W. Newman, A. K. Singh, J. J. Venit, C. J. Spagnuolo, A. T. M. Serajuddin, Int. J. Pharm. 1994, 105, 209-217.

[6] R. K. Khankari, D. J. W. Grant, Thermochim. Acta 1995, 248, 61 79.

[7] U. J. Griesser, in Polymorphism (Ed.: R. Hilfiker), Wiley-VCH, Weinheim, Germany, 2006, pp. 211-233.

[8] S. R. Byrn, W. Xu, A. W. Newman, Adv. Drug Delivery Rev. 2001, 48, 115-136.

[9] a K. R. Morris, in Polymorphism in Pharmaceutical Solids, Vol. 95 (Ed.: H. G. Brittain), Marcel Dekker Ltd, New York, 1999, pp. 125181; b K. Fucke, J. W. Steed, Water 2010, 2, 333-350.

[10] a S. N. Ivashevskaya, J. van de Streek, J. E. Djanhan, J. Bruening E. Alig, M. Bolte, M. U. Schmidt, P. Blaschka, H. W. Hoeffken, P. Erk, Acta Cryst. B 2009, B65, 212-222; b R. T. Forbes, P. York, V. Fawcett, L. Shields, Pharm. Res. 1992, 9, 1428-1435.

[11] G. A. Stephenson, A. Aburub, T. A. Woods, J. Pharm. Sci. 2011 100, 1607-1617.

[12] L. H. Nielsen, S. Gordon, J. P. Pajander, J. Østergaard, T. Rades, A. Müllertz, Int. J. Pharm. 2013, 457, 14-24.

[13] A. Serajuddin, A. B. Thakur, R. N. Ghoshal, M. G. Fakes, S. A Ranadive, K. R. Morris, S. A. Varia, J. Pharm. Sci. 1999, 88, 696704.

[14] N. P. A. Christensen, S. Nielsen, J. Rantanen, C. Cornett, P. Bertelsen, Powder Technol. 2014, 266, 175-182.

[15] P. Tong, G. Zografi, J. Pharm. Sci. 2001, 90, 1991-2004.

[16] a B. C. Hancock, M. Parks, Pharm. Res. 2000, 17, 397-404; b S. B. Murdande, M. J. Pikal, R. M. Shanker, R. H. Bogner, J. Pharm. Sci. 2010, 99, 1254-1264.

[17] M. Bartolomei, P. Bertocchi, E. Antoniella, A. Rodomonte, J. Pharm. Biomed. Anal. 2006, 40, 1105-1113. Pharmacopoeia Europaea 9.0, Deutscher Apotheker Verlag, Stuttgart \& Govi-Verlag GmbH, Eschborn, 2016.

[19] M. Ono, H. Anzai, Y. Tozuka, K. Moribe, T. Oguchi, K. Yamamoto,
Chem. Pharm. Bull. 2005, 53, 180-183.

[19] M. Ono, H. Anzai, Y. Tozuka, K. Moribe, T. Oguchi, K. Yamamoto,
Chem. Pharm. Bull. 2005, 53, 180-183. Y. Xu, L. Jiang, Y. Huang, J.-R. Wang, X. Mei, J. Pharm. Sci. 2014, 103, 3688-3695.

[21] L. R. Chen, V. G. Young Jr, D. Lechuga-Ballesteros, D. J. W. Grant, J. Pharm. Sci. 1999, 88, 1191-1200. 
[22] D. Gibbs, A. Jackman, P. Kirkpatrick, Nature rev. Drug disc. 2005, 4, S16.

[23] R. Khankari, L. Chen, D. J. Grant, J. Pharm. Sci. 1998, 87, 1052 1061.

[24] G. A. Stephenson, B. A. Diseroad, Int. J. Pharm. 2000, 198, 167177.

[25] M. Bartolomei, A. Rodomonte, E. Antoniella, G. Minelli, P. Bertocchi, J. Pharm. Biomed. Anal. 2007, 45, 443-449.

[26] L. Malaj, R. Censi, P. D. Martino, Cryst. Growth Des. 2009, 9, 2128-2136.

[27] United States Pharmacopoeia Vol 40, The Stationary Office, Norwich, England, 2017.

[28] a K. Fucke, J. A. K. Howard, J. W. Steed, Chem. Commun. 2012, 48, 12065-12067; b K. Fucke, G. J. McIntyre, M.-H. Lemée-
Cailleau, C. Wilkinson, A. J. Edwards, J. A. K. Howard, J. W. Steed, Chem. Eur. J. 2015, 21, 1036-1047.
[29]

[30]

[31] K. Fucke, M. J. G. Peach, J. A. K. Howard, J. W. Steed, Chem. commun. 2012, 48, 9822-9824.

L. E. Tonnessen, B. F. Pedersen, J. Klaveness, Acta Chem. Scand. 1996, 50, 603-608.

K. Fucke, G. J. McIntyre, C. Wilkinson, M. Henry, J. A. K. Howard, J. W. Steed, Cryst. Growth Des. 2012, 12, 1395-1401.

D. E. Braun, U. J. Griesser, Cryst. Growth Des. 2016, 16, 61116121

O. V. Dolomanov, L. J. Bourhis, R. J. Gildea, J. A. K. Howard, H. Puschmann, J. Appl. Crystallogr. 2009, 42, 339-341. 


\section{Entry for the Table of Contents}

\section{FULL PAPER}

The pharmaceutical X-ray contrast diatrizoic acid sodium salt can exist in at least five hydrated crystal forms containing between 0.3 and 8 molar equivalents of water. The hydrates interconvert depending on the relative humidity. Of the two found solvates, one contains water in addition to DMSO. This compound is a valuable model for water affinity of sodium salts in the solid state.

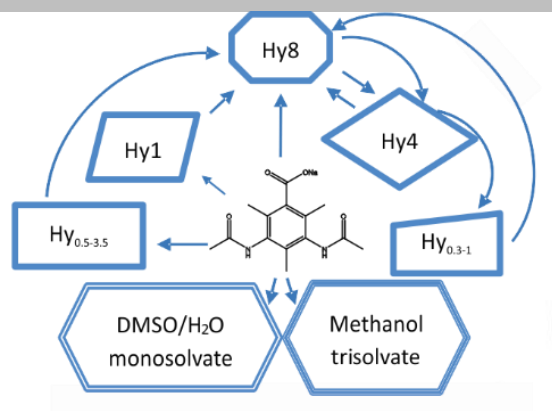

Mohd Nadzri Mohd Najib, Kevin Back Katharina Edkins*

Page No. - Page No.

The Complex Solid-State Landscape of Sodium Diatrizoate Hydrates 